> Les épithéliums qui bordent le tubule contourné proximal, le feuillet viscéral du sac vitellin et l'iléon ont la capacité d'internaliser par endocytose une grande variété de composants, notamment protéiques, qui permet leur approvisionnement en composés cruciaux, mais aussi le transport sélectif de composés d'un compartiment vers un autre. Un récepteur multiligands, la cubiline, en association avec la mégaline, un autre récepteur multiligands, joue un rôle crucial dans ces processus, en physiologie comme en pathologie. <

\section{Cubiline : rôle physiopathologique et relations avec la mégaline}

Pierre J. Verroust, Renata Kozyraki

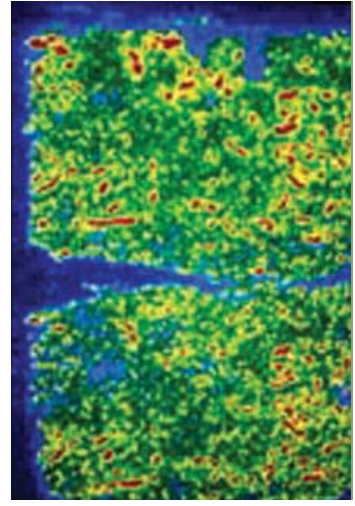

rent le recyclage de la membrane plasmique apicale internalisée [2]. Deux récepteurs multiligands semblent constituer le support biochimique de cette activité, la mégaline, ou gp330, et la cubiline, encore appelée gp280 ou récepteur du complexe facteur intrinsèque gastrique/cobalamine (IFCR) $[3,4]$. Ces protéines ne sont exprimées que dans un nombre restreint d'épithéliums, dont le TCP, le feuillet viscéral du sac vitellin et l'iléon terminal, où elles sont concentrées dans les puits à clathrine. Bien que de structure très différente, elles sont toutes deux caractérisées par l'accumulation de domaines de liaison qui leur confèrent des propriétés de récepteurs multiligands. Elles sont étroitement coexprimées et interagissent entre elles, mais servent des fonctions distinctes dans les différents organes où elles sont exprimées. Dans cet article, nous présentons brièvement les propriétés de la cubiline, son importance fonctionnelle et ses relations avec la mégaline.

\section{Structure de la cubiline}

La cubiline [5-7] est une protéine périphérique de 460 $\mathrm{kDa}$, contenant 13 à $14 \%$ d'hydrates de carbone et dont 
la séquence primaire, déterminée chez le rat, l'homme et le chien est conservée avec une homologie de $70 \%$. La structure de la cubiline (Figure 1) montre une séquence amino-terminale de 110 acides aminés, suivie d'un assemblage de 35 modules extracellulaires comportant 8 domaines EGF (epidermal growth factor) de type B et 27 domaines CUB (complément $\mathrm{Cl} / \mathrm{Cl}$ s, Uegf [epidermal growth factor sea urchin related protein] et bone morphogenic protein-1) ayant entre eux une homologie globale de $40 \%$. La cubiline ne contient pas de domaine transmembranaire ni d'ancre glycosylphosphatidylinositol. Son association au versant externe de la membrane plasmique [8] implique probablement les 100 résidus amino-terminaux. Cette région comporte une cystéine libre qui pourrait être palmitoylée [9] et une région susceptible de former une hélice amphipathique $\alpha$ qui ressemble au domaine de liaison des apolipoprotéines aux lipides. L'élément le plus frappant dans la structure de la cubiline est l'accumulation de 27 domaines CUB qui lui confère la possibilité de se lier à une grande variété de ligands (Tableau 1). Le site de liaison du complexe facteur intrinsèque gastrique/vitamine B12 a été localisé dans le domaine CUB8. Les autres ligands identifiés semblent être reconnus par des domaines CUB plus proches de l'extrémité carboxy-terminale [8]. L'un de ces ligands est la mégaline, un autre récepteur multiligand co-localisé avec la cubiline (Figures 2 à 4) dont nous verrons qu'il facilite l'internalisation.

\section{Expression de la cubiline}

Les quantités les plus importantes de cubiline sont retrouvées dans le TCP, l'épithélium viscéral du sac vitellin, l'iléon et la muqueuse utérine [10]. À l'échelon subcellulaire, la cubiline est étroitement co-localisée avec la mégaline [6]. Les deux récepteurs sont décelables dans l'ensemble de l'appareil endocytique apical (EIMV/puits à clathrine, endosomes, tubules apicaux denses) et à un moindre degré dans les lysosomes.

La biosynthèse de la cubiline est complexe et inhabituelle [11]: elle serait adressée à la membrane plasmique sous forme immature, sensible à l'endoglycosidase $H$, recyclée vers l'appareil de Golgi et ré-adressée à la membrane plasmique après maturation des chaînes osidiques. Alors que l'association avec la RAP (receptor associated protein), une protéine d'escorte impliquée dans la biosynthèse des récepteurs de la famille du low density lipoprotein receptor, est indispensable à l'expression membranaire de la mégaline, elle ne semble pas nécessaire à l'expression de la cubiline qui pourrait, en revanche, nécessiter l'intervention d'une autre protéine d'escorte encore non identifiée $[7,12]$.

\section{Ligands et fonctions de la cubiline}

Les principaux ligands de la cubiline et de son co-récepteur, la mégaline, sont présentés dans le Tableau I. La cubiline peut se lier à plusieurs ligands simultanément. La formation des complexes cubiline-ligand nécessite la présence de calcium. L'internalisation de ces complexes fait intervenir au moins un co-récepteur, la mégaline. Cette conclusion repose sur l'interaction démontrée entre cubiline et mégaline et la co-localisation étroite des deux récepteurs [6], l'effet inhibiteur des anticorps anti-mégaline sur l'internalisation de la plupart des ligands, et l'absence d'internalisation des complexes ligand-cubiline chez les souris mégaline $e^{-/-}$[13-16].

Le rôle physiologique de la cubiline varie en fonction du site d'expression considéré. La cubiline est le récepteur des complexes facteur intrinsèque-vitamineB12 $\left(\mathrm{Fl}_{12} \mathrm{~B}_{12}\right)$

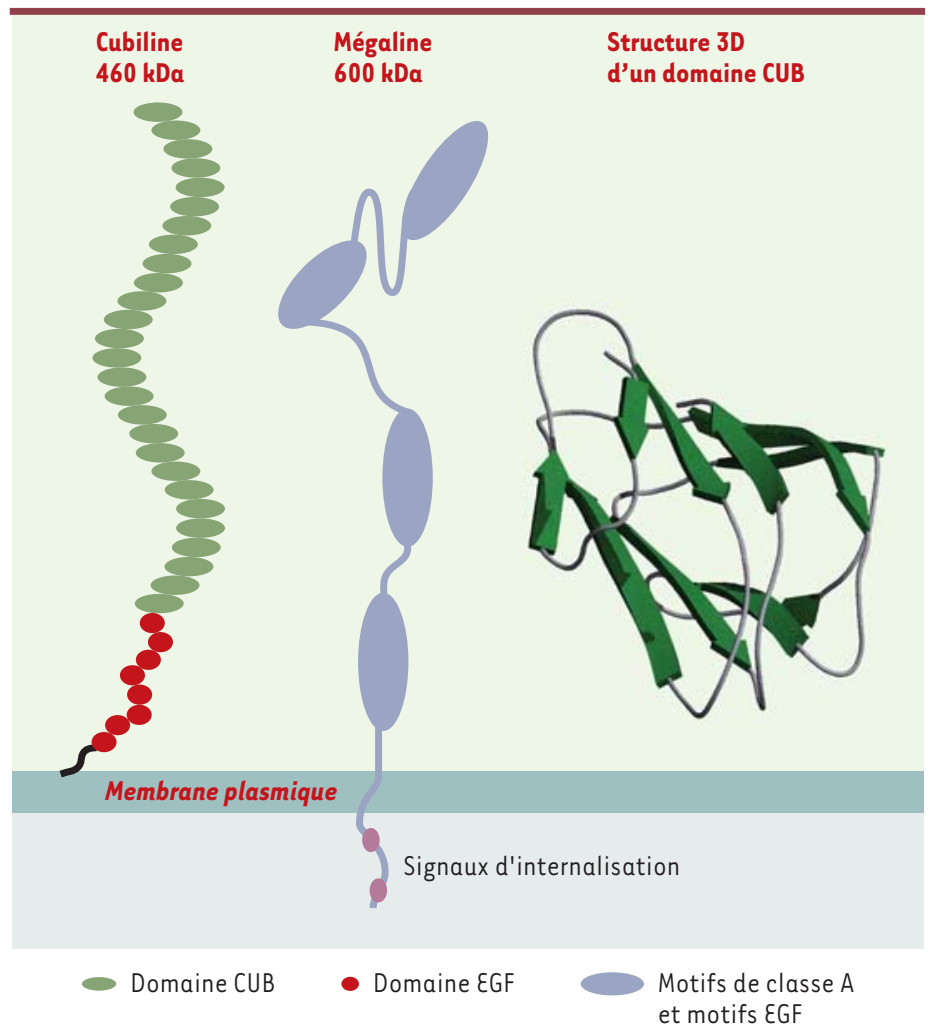

Figure 1. Représentation schématique de la cubiline et de la mégaline. La structure tridimensionnelle d'un domaine CUB (complément $\mathrm{Cl} / \mathrm{Cl}$ s, Uegf [epidermal growth factor sea urchin related protein] et bone morphogenic protein-1), caractérisée par deux couches de 5 feuillets $\beta$ antiparallèles reliés par des coudes $\beta$, a été déterminée en prenant comme matrice la structure des spermadhésines. La mégaline est caractérisée par trente-six motifs de classe A ou ligand binding repeats, flanqués de motifs EGF (epidermal growth factor) et répartis en quatre blocs. Le domaine cytoplasmique comporte deux signaux d'internalisation par les puits à clathrine du type NPXY (Asn-Pro-X-Tyr). 
indispensable à l'absorption physiologique de cette vitamine dans l'iléon; elle intervient dans la réabsorption des protéines par le TCP et joue un rôle clé au cours du développement. Elle pourrait avoir d'autres fonctions, notamment dans le métabolisme du cholestérol.

\section{La cubiline: récepteur physiologique}

du complexe facteur intrinsèque/vitamine B12

L'absorption intestinale de la vitamine B12 (Figure 2) implique la formation d'un complexe entre la vitamine B12 alimentaire et une protéine sécrétée par la muqueuse gastrique, le facteur intrinsèque ( $\mathrm{FI})$. Ce complexe est lui-même internalisé par un récepteur de haute affinité exprimé au pôle apical des entérocytes de l'iléon terminal. Après internalisation, le Fl est dégradé tandis que la vitamine B12 est sécrétée dans les capillaires au pôle basal de la cellule iléale. La mise en évidence de ce récepteur de forte affinité dans les cellules épithéliales du TCP [3] et le sac vitellin nous a amenés à montrer qu'il était identique à la gp280, une protéine que nous avions identifiée et caractérisée comme cible d'anticorps tératogènes [4]. L'étude de patients ayant un déficit héréditaire de l'absorption de la vitamine B12, connu sous le nom d'anémie mégaloblastique autosomique récessive ou syndrome d'Imerslund-Gräsbeck (SIG), a permis d'acquérir la certitude que la cubiline était le récepteur physiologique de ce complexe. En effet, le gène de la cubiline est situé sur le bras court du chromosome 10 (10p12.33-p13) dans une région de $6 \mathrm{~cm}$ contenant MGAl (megaloblastic anemia-1), le locus présomptif du SIG. La structure de la cubiline est anormale chez ces patients [17].

\section{CUBILINE}

\section{Transporteurs sériques}

Facteur intrinsèque gastrique/vitamine $B_{12}$

Transferrine/Fer

DBP (vitamin D-binding protein)/vitamine D

\section{Lipoprotéines}

Apolipoprotéine Al (ApoAl) et HDL (high density lipoprotein)

\section{Autres protéines}

Albumine

Receptor associated protein

Chaînes légères des immunoglobulines

Hémoglobine

Clara cell secretory protein

\section{Médicaments ou toxines}

Aminoglycosides

Tableau I. Principaux ligands de la cubiline et de la mégaline.

\section{MÉGALINE}

Retinol binding protein/vitamine $A$

Transcobalamine II/vitamine $B_{12}$

DBP/vitamine D

Apo B, Apo E, Apo J

Aminoglycosides

Trichosantine

\section{Albumine}

Receptor associated protein

Chaînes légères des immunoglobulines Hémoglobine

Seize des dix-sept patients étudiés ont une mutation ponctuelle qui substitue une proline à une leucine (FMI u finnish mutation 1) dans le domaine CUB8 qui porte I'un des introns du domaine CUB6 et conduisent à la Cubiline et réabsorption des protéines dans le TCP Bien que la cubiline exprimée par le TCP et l'épithélium vitellin soit capable expérimentalement de lier et d'internaliser le complexe FI/B12, il est probable que cette propriété n'est pas physiologiquement importante car le FI n'est présent qu'en quantité très faible dans la circulation et donc dans le TCP. L'existence d'une protéinurie caractérisée par la présence, dans l'urine, de en normalement indécelables en aval du TCP chez de protéines présentes dans le TCP (Figure 3).

政 la DBP (vitamin D-binding protein) ou la CCSP (Clara cell secretory protein) et l'absence de vacuoles de réabsorption contenant ces mêmes protéines [14, 15, 18] dans le TCP des chiens ayant un déficit d'adressage membranaire de la cubiline. Ces vacuoles sont en revanche facilement décelables chez les temoins. La réabsorption des protéines par le TCP implique en 列 internalisation des complexes cubiline-ligand. Pour d'autres protéines, notamment l'albumine et la DBP (vitamin D-binding protein), la cubiline, mais aussi la mégaline, sont responsables de la liaison apicale. Enfin certaines protéines, telles que la TC II (transcobalamine II) ou la retinol binding protein ne sont pas reconnues par la cubiline et sont réabsorbées par la mégaline. L'expression abondante de la cubiline et de la mégaline tout au long du TCP, l'efficacité de leur recyclage par le système des tubules apicaux den- 
ses et la localisation dans les segments précoces du TCP de la plupart de leurs ligands suggèrent que les capacités de réabsorption protéique que constitue le complexe cubiline/mégaline ne sont pas saturées dans les conditions normales. Ce système permet la réabsorption de plusieurs grammes de protéines par jour, contribuant ainsi à l'équilibre du bilan azoté. Lorsque la perméabilité glomérulaire est anormalement élevée, la capacité de réabsorption induit un trafic protéique accru dans les cellules tubulaires proximales, dont il est généralement admis qu'il constitue la première étape vers le développement de la sclérose rénale et la progression vers l'insuffisance rénale [19].

Le tandem cubiline/mégaline permet également de préserver certains éléments essentiels tels que le fer et les vitamines $B 12$ [20], A et $D$. Les complexes formés par la vitamine $D$ et la DBP (une protéine de $58 \mathrm{kDa}$ ), présents dans la lumière du TCP, sont internalisés et adressés dans les lysosomes qui dégradent la DBP tandis que la vitamine $D$ est transférée dans le cytosol, hydroxylée dans les mitochondries et sécrétée au pôle baso-latéral, probablement via un système vésiculaire qui reste à identifier (Figure 3). L'importance physiologique de cette voie d'internalisation est démontrée par les anomalies majeures du système osseux observées chez les souris mégaline $e^{-/-}$ [21].

\section{Cubiline et développement fœtal}

La cubiline est exprimée très précocement au cours du développement embryonnaire chez les rongeurs. Elle est décelée dès J4-

Figure 2. Schéma illustrant la place de la cubiline et de la mégaline dans l'absorption intestinale et le métabolisme de la vitamine B12. Le complexe formé par la vitamine B12 présente dans les aliments et le facteur intrinsèque $(\mathrm{FI})$ gastrique est internalisé par la cubiline co-exprimée avec la mégaline au pôle apical des entérocytes de l'iléon terminal. Le facteur intrinsèque est dégradé tandis que la vitamine B12 est transférée dans la circulation sous forme complexée à la transcobalamine II (TCII). Le mécanisme, et notamment le système vésiculaire éventuel, impliqué dans la formation de ce complexe est inconnu. Les complexes transcobalamine/vitamine B12 sont internalisés dans le foie et les organes périphériques par un récepteur ubiquitaire dont l'identification est encore incertaine. Le cas du rein est particulier, car la mégaline exprimée au pôle apical des cellules du TCP permet l'internalisation des complexes TCII-B12 présents dans le fluide tubulaire, et la sauvegarde de quantités importantes de vitamine B12, probablement du même ordre de grandeur que les quantités absorbées dans l'intestin.
J5 (Figure 4) dans les cellules de l'endoderme primitif qui migrent, à partir de la masse cellulaire interne, pour tapisser la cavité blastocœlique. Son expression persiste sur le feuillet viscéral du sac vitellin jusqu'au terme de la gestation. Pendant une période allant de $J 4$ à $J 9$, ce feuillet constitue l'interface fonctionnelle unique entre la mère et le fœtus. L'épithélium pariétal est perméable aux protéines maternelles qui ont ainsi accès à la cavité vitelline. Selon les conceptions physiologiques actuelles, la cubiline exprimée au pôle apical des cellules viscérales (Figure 4) permet, en association avec la mégaline, l'internalisation puis la dégradation des protéines et des lipoprotéines maternelles présentes dans la cavité vitelline. Les acides aminés issus de ce processus sont transférés vers l'embryon, tandis que le cholestérol (Figure 4) est reconditionné dans de nouvelles particules lipoprotéiques formées à partir de lipoprotéines Al et/ou B synthétisées par les cellules vitellines et sécrétées dans les capillaires vitellins. Un mécanisme similaire interviendrait pour le transfert du fer.

L'importance de la cubiline au cours du développement fœtal a été, dans un premier temps, mise en évidence par le pouvoir tératogène chez le rat des anticorps anticubiline administrés au $9^{e}$ jour de la gestation. Ces

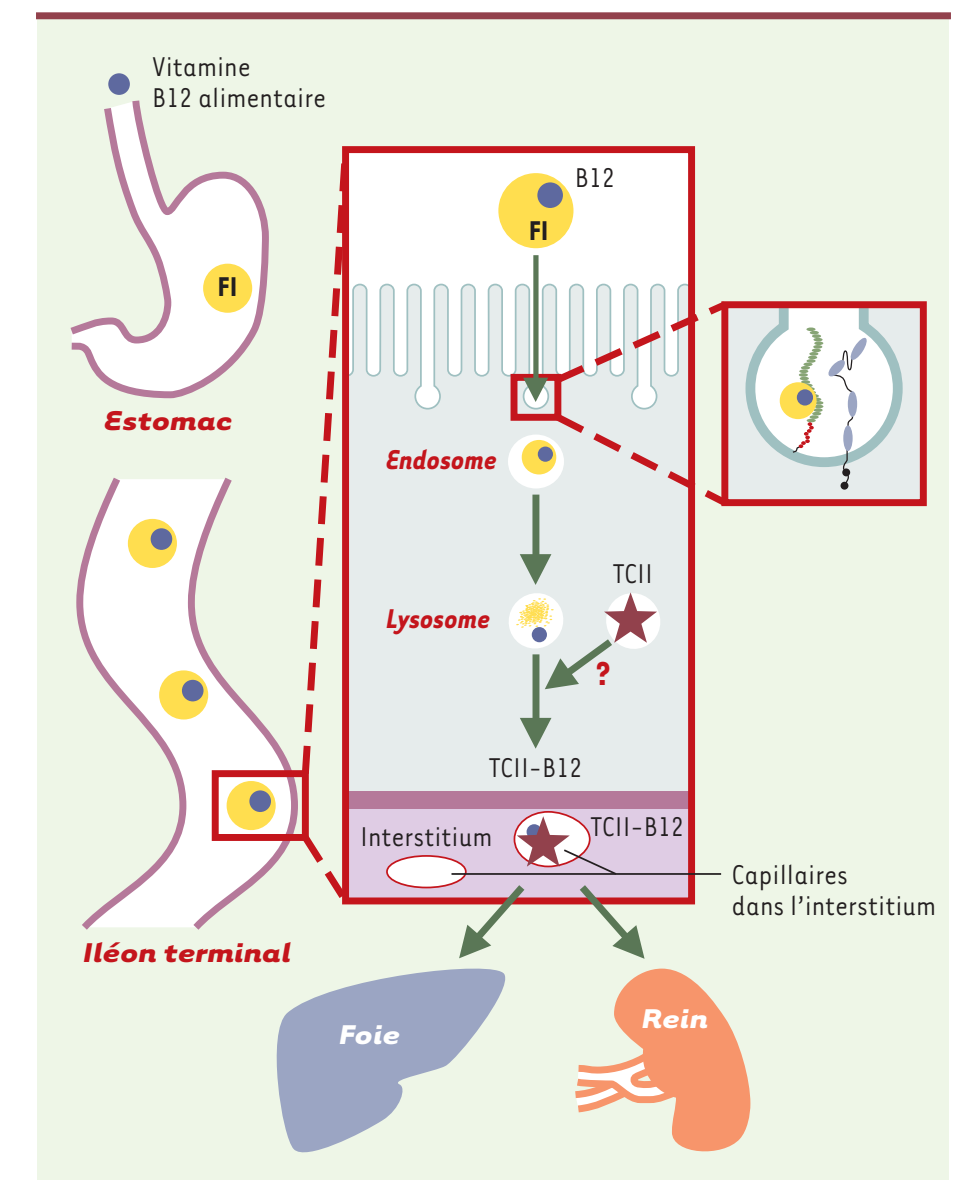


résultats peuvent être rapportés à la fixation des anticorps sur le sac vitellin et à une inhibition des mécanismes d'endocytose $[22,23]$ qui assurent les transferts de la mère vers le fœtus (Figure 4). L'effet des anticorps anti-cubiline pourrait être expliqué par une diminution de la quantité de protéines internalisées, ce qui conduirait à une diminution de la quantité d'acides aminés transférés vers l'embryon. Il pourrait aussi être dû à une inhibition du transfert de cholestérol de la mère vers l'embryon, comme le suggère la similitude des malformations induites par les anticorps anti-cubiline et les inhibiteurs de synthèse du cholestérol.

Le rôle de la cubiline au cours du développement est probablement plus complexe, car les premières expé-

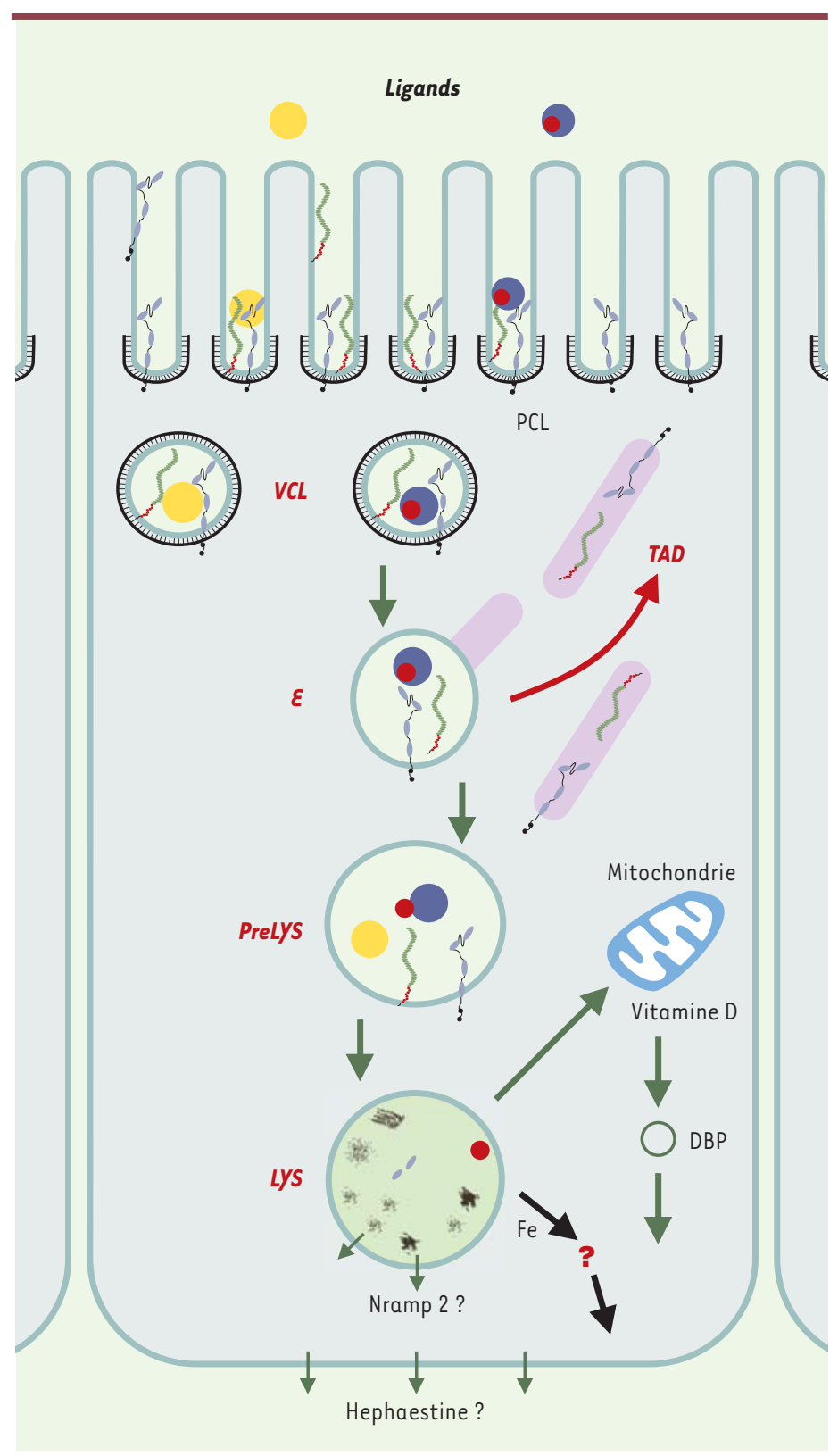

riences d'invalidation du gène de la cubiline indiquent que les embryons meurent très précocement in utero. L'expression de la cubiline par les toutes premières cellules de l'endoderme primitif pourrait être indispensable au développement ultérieur de l'embryon [24]. De plus, les données récentes obtenues dans notre laboratoire indiquent que la cubiline est co-exprimée avec la mégaline dès les premiers stades de développement du neuroépithélium, et pourrait jouer un rôle dans ce contexte. Les anomalies du développement, et notamment le syndrome holoprosencéphalique chez les souris mégaline ${ }^{-/-}$ [25] (qui ont une invalidation fonctionnelle au moins partielle du gène de la cubiline) vont dans ce sens.

\section{Autres fonctions de la cubiline}

Comme nous l'avons indiqué plus haut, la cubiline se lie aux HDL (high densitiy lipoprotein) avec une forte affinité et permet, avec l'aide de la mégaline, d'internaliser l'ensemble de la particule. Au cours de ce processus, I'apoA-I est

Figure 3. Schéma illustrant la fonction de la cubiline dans l'endocytose par les cellules tubulaires proximales. La cubiline est exprimée au pôle apical des cellules épithéliales, dans la bordure en brosse et plus particulièrement dans les espaces intermicrovillaires. Les ligands fixés par la cubiline sont internalisés dans les puits à clathrine $(\mathrm{PCl})$ qui se séparent de la membrane pour former des vésicules recouvertes de clathrine (VCI). Ce processus implique très probablement l'association de la cubiline à un co-récepteur tel que la mégaline qui porte des signaux d'internalisation caractéristiques de la famille du LDLR (low density lipoprotein receptor). Les $\mathrm{VCl}$ se transforment en endosomes $(\varepsilon)$ de taille variable dans lesquels commence, sous l'effet $\mathrm{du} \mathrm{pH}$ acide, la dissociation ligand/récepteur. Les tubules apicaux denses (TAD) assurent le recyclage de la cubiline et de la mégaline. Il est possible que le recyclage ne soit pas exhaustif dans la mesure où elles peuvent être décelées dans la matrice lysosomale. Les ligands sont transférés vers les prélysosomes (PreLyS), puis les lysosomes (LYS) où ils sont dégradés. Lorsque le ligand de la cubiline est lui-même un complexe entre une protéine de liaison et un ligand (exemple: DBP ou vitamin D-binding protein et vitamine $D$, transferrine et fer) symbolisé ici en bleu et rouge, l'ensemble du complexe est internalisé et acheminé vers les lysosomes qui dégradent la protéine de liaison et libèrent le ligand. Les mécanismes qui permettent la sortie des ligands des lysosomes sont très mal connus et probablement variables en fonction du ligand. Le système vésiculaire potentiellement impliqué n'est pas clairement identifié. Les transporteurs du fer (Nramp2 ou natural resistance-associated macrophage protein 2 et Hephaestine?) sont présents dans la paroi des lysosomes et au pôle basolatéral des cellules tubulaires. 
dégradée [13]. Le mécanisme d'endocytose des lipides par la cubiline, distinct de l'internalisation sélective du cholestérol par le scavenger receptor $B$ I, se rapproche donc du mécanisme décrit par Brown et Goldstein pour les LDL (low densitiy lipoprotein). L'importance physiologique de cette propriété n'est pas entièrement élucidée. La cubiline intervient dans la réabsorption de I'apoA-I par le TCP. À l'état normal, I'apoA-I est filtrée, réabsorbée puis dégradée dans les cellules du TCP, et n'est pas décelable dans l'urine. II est possible que la cubiline intervienne de manière plus générale dans le métabolisme des HDL.

\section{Conclusions et perspectives}

Les données brièvement présentées dans cet article montrent que la cubiline, en association avec la mégaline, joue un rôle important dans l'absorption intestinale de la vitamine B12, la réabsorption des protéines par les cellules épithéliales du TCP, les échanges mère-fœtus et, de manière plus générale, dans le développement embryonnaire. Ces observations ouvrent des perspectives physiopathologiques intéressantes dans plusieurs domaines. L'élucidation du rôle de la cubiline au cours du développement constitue une voie d'investigation particulièrement passionnante puisque la majorité des malformations fœtales en pathologie humaine sont d'origine inconnue. Par ailleurs, l'identification du couple cubiline/mégaline permet d'envisager le développement rationnel d'agents susceptibles de limiter la réabsorption des protéines ou de toxiques (aminoglycosides, par exemple) et donc de limiter les conséquences néfastes qui peuvent en résulter, telles que la fibrose rénale ou la toxicité tubulaire proximale. Cette approche passe par une dissection fonctionnelle approfondie de la cubiline, permettant d'identifier les sites de liaison, les mécanismes d'internalisation et le trafic du récepteur. $\diamond$

\section{SUMMARY}

Physiopathology of cubilin and

its interactions with megalin

Epithelia which line the renal proximal convoluted tubule, the visceral layer of the yolk sac and the ileum have the ability to internalize a variety of substances which not only serve as nutrients, but may also be transported from one compartment to another. Cubilin, a multiligand receptor, in association with megalin, also a multiligand receptor, appears to be important under both normal and pathological conditions. $\Delta$

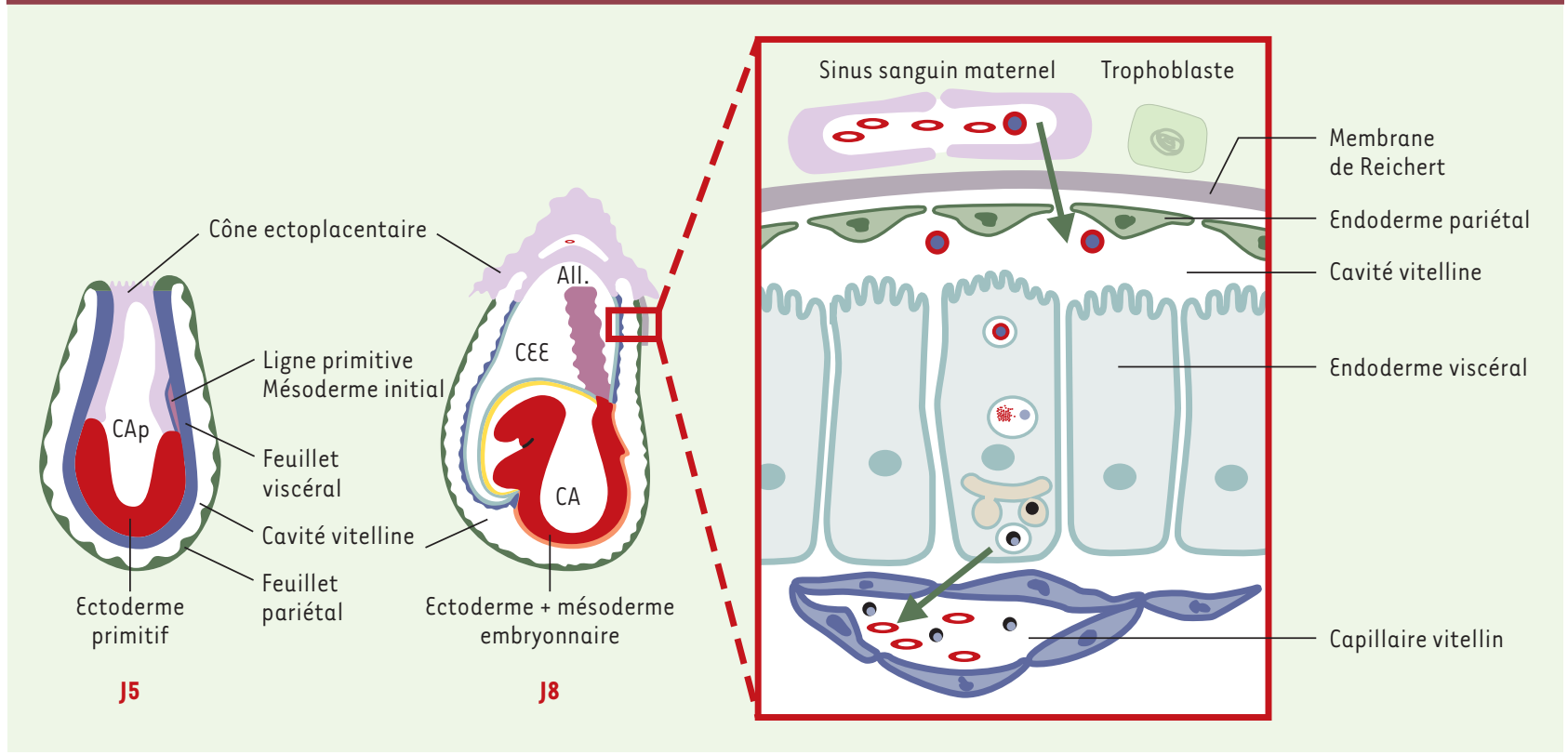

Figure 4. Place de la cubiline dans la fonction du sac vitellin. La cubiline est exprimée au pôle apical des cellules épithéliales qui bordent le feuillet viscéral du sac vitellin dès J4-J5. Entre $J 8$ et J11, le feuillet viscéral (en bleu) du sac vitellin constitue la seule interface fonctionnelle entre la mère et le fœtus, jusqu'à la mise en place du placenta allantoïdien (All.). cદદ: cœlome extra-embryonnaire; CA(p): cavité amniotique (primitive). Chez les rongeurs, les HDL (high density lipoprotein) (cercle rouge entourant un point bleu) sont internalisées au pôle apical des cellules vitellines par l'intermédiaire du complexe cubiline/mégaline. L'apolipoprotéine Al est dégradée, tandis que le cholestérol (point bleu) est reconditionné dans de nouvelles particules lipoprotéiques formées à partir d'apolipoprotéines Al et B synthétisées par les cellules vitellines. 


\section{RÉFÉRENCES}

1. Jollie WP. Development, morphology, and function of the Yolk-Sac placenta of laboratory rodents. Teratology 1990; 41 : 361-81.

2. Christensen $\varepsilon I$, Birn $\mathrm{H}$, Verroust $P$, et al. Membrane receptors for endocytosis in renal proximal tubule. Int Rev Cytol 1998; 180 : 237-84.

3. Seetharam B, Levine JS, Ramasamy $M$, et al. Purification, properties, and immunochemical localization of a receptor for intrinsic factorcobalamin complex in the rat kidney. J Biol Chem 1988; 263: 4443-9.

4. Seetharam B, Christensen El, Moestrup SK, et al. Identification of rat yolk sac target protein of teratogenic antibodies, gp280, as intrinsic factorcobalamin receptor. J Clin Invest 1997; 99: 2317-22.

5. Kozyraki R, Kristiansen M, Silahtaroglu $A$, et al. The human intrinsic factorvitamin B12 receptor, cubilin: molecular characterization and chromosomal mapping of the gene to $10 p$ within the autosomal recessive megaloblastic anemia (MGAl) region. Blood 1998 ; 91: 3593-600.

6. Moestrup SK, Kozyraki R, Kristiansen $M$, et al. The intrinsic factor-vitamin $B 12$ receptor and target of teratogenic antibodies is a megalin-binding peripheral membrane protein with homology to developmental proteins. J Biol Chem 1998; 273: 5235-42.

7. Xu D, Kozyraki R, Newman TC, et al. Genetic evidence of an accessory activity required specifically for cubilin brush-border expression and intrinsic factor-cobalamin absorption. Blood 1999; 94: 3604-6.

8. Kristiansen $M$, Kozyraki R, Jacobsen C, et al. Molecular dissection of the intrinsic factor-vitamin B12 receptor, cubilin, discloses regions important for membrane association and ligand binding. J Biol Chem 1999; 274 : 20540-4.

9. Ramanujam KS, Seetharam S, Dahms NM, et al. Effect of processing inhibitors on cobalamin (vitamin B12) transcytosis in polarized opossum kidney cells. Arch Biochem Biophys 1994; 315: 8-15.

10. Sahali D, Mulliez N, Chatelet $\mathrm{F}$, et al. Comparative immunochemistry and ontogeny of two closely related coated pit proteins. The 280-kd target of teratogenic antibodies and the 330-kd target of nephritogenic antibodies. Am J Pathol 1993; 142 : 1654-67.

11. Baricault L, Galceran M, Ronco PM, et al. Unusual processing of GP280, a protein associated with the intermicrovillar areas of yolk sac epithelial cells: plasma membrane delivery of immature protein. Biochem Biophys Res Commun 1995; 212 : 353-9.

12. Fyfe JC, Ramanujam KS, Ramaswamy K, et al. Defective brush-border expression of intrinsic factor-cobalamin receptor in canine inherited intestinal cobalamin malabsorption. J Biol Chem 1991; 266 : 4489-94.

13. Kozyraki R, Fyfe J, Kristiansen $M$, et al. The intrinsic factor-vitamin $\mathrm{B} 12$ receptor, cubilin, is a high-affinity apolipoprotein $A-I$ receptor facilitating endocytosis of high-density lipoprotein. Nat Med 1999; 5: 656-61.
14. Kozyraki R, Fyfe J, Verroust PJ, et al. Megalindependent cubilinmediated endocytosis is a major pathway for the apical uptake of transferrin in polarized epithelia. Proc Natl Acad Sci USA 2001; 98: 12491-6.

15. Nykjaer A, Fyfe JC, Kozyraki $R$, et al. Cubilin dysfunction causes abnormal metabolism of the steroid hormone $25(\mathrm{OH})$ vitamin D(3). Proc Natl Acad Sci USA 2001; 98: 13895-900.

16. Burmeister $R$, Boe IM, Nykjaer A, et al. A tworeceptor pathway for catabolism of Clara cell secretory protein in the kidney. J Biol Chem 2001; 276: 13295-301.

17. Aminoff $M$, Carter $J \varepsilon$, Chadwick RB, et al. Mutations in CUBN, encoding the intrinsic factor-vitamin B12 receptor, cubilin, cause hereditary megaloblastic anaemia 1. Nat Genet 1999; 21 : 309-13.

18. Birn H, Fyfe JC, Jacobsen C, et al. Cubilin is an albumin binding protein important for renal tubular albumin reabsorption. J Clin Invest 2000; 105: 1353-61.

19. Ruggenenti P, Remuzzi G. The role of protein traffic in the progression of renal diseases. Annu Rev Med 2000 ; 51: 315-27.

20. Moestrup SK, Birn H, Fischer $\mathrm{PB}$, et al. Megalinmediated endocytosis of transcobalamin-vitaminB12 complexes suggests a role of the receptor in vitamin-B12 homeostasis. Proc Natl Acad Sci USA 1996; 93 : 8612-7.
21. Nykjaer A, Dragun D, Walther D, et al. An endocytic pathway essential for renal uptake and activation of the steroid $25-(\mathrm{OH})$ vitamin D3. Cell 1999; 96: 507-15.

22. Sahali D, Mulliez N, Chatelet $\mathrm{F}$, et al.

Characterization of a 280 $\mathrm{kD}$ protein restricted to the coated pits of the renal brush border and the epithelial cells of the yolk sac. Teratogenic effect of the specific monoclonal antibodies. J Exp Med 1988 ; 167: 213-8.

23. Le Panse $S$, Ayani $\varepsilon$, Mulliez $N$, et al. Antibodies to the 280-kD coated pit protein, target of teratogenic antibodies, produce alterations in the traffic of internalized proteins. Am J Pathol 1994; 145: 1526-36.

24. Beddington RS, Robertson ह). Axis development and early asymmetry in mammals. Cell 1999; 96 : 195-209.

25. Willnow TE, Hilpert J, Armstrong SA, et al. Defective forebrain development in mice lacking gp330/megalin. Proc Natl Acad Sci USA 1996; $93: 8460-4$. 